\title{
L'indice di Flugelman per individuare pazienti complessi e di difficile dimissione
}

\author{
Use of the Flugelman index for identifying patients who are difficult \\ to discharge from the hospital
}

\section{Chiara Bozzano ${ }^{a, *}$, llario Lancini ${ }^{a}$, Elena Mei $^{a}$, Maida Lucarini ${ }^{\mathrm{b}}$, Roberta Mastriforti ${ }^{b}$, Nunzia Zuccone ${ }^{a}$, Dino Vanni ${ }^{a}$, Claudio Pedace ${ }^{c}$}

${ }^{a}$ UOC Medicina Interna, Ospedale San Donato, Azienda USL 8 Arezzo

${ }^{\mathrm{b}}$ UOC Medicina Interna, Ospedale Valtiberina, Azienda USL 8 Arezzo

${ }^{\mathrm{C}}$ Distretto Zona Aretina, Azienda USL 8 Arezzo

Ricevuto il 29 aprile 2010; accettato il 14 giugno 2010

disponibile online il 25 novembre 2010

\section{KEYWORDS \\ Difficult hospital \\ discharge; \\ Flugelman index; \\ Multidimensional \\ assessment.}

\begin{abstract}
Summary
Introduction: To evaluate the use of multidimensional assessment based on the Fluegelman Index (FI) to identify internal medicine patients who are likely to be difficult to discharge from the hospital.

Materials and methods: Have been evaluated all patients admitted to the medical wards of the District General Hospital of Arezzo from September 1 to October 31, 2007. We collected data on age, sex, socioeconomic condition, cause of admission, comorbidity score preadmission functional status (Barthel Index), incontinence, feeding problems, length of hospitalization, condition at discharge, and type of discharge. The FI cut off for difficult discharge was $\geq 17$.

Results: Of the 413 patients (mean age $80 \pm 11.37$ years; percentage of women, $56.1 \%$ ) included in the study, 109 (26.39\%) had Flugelman Index $\geq 17$. These patients were significantly older than the patients with lower Fls ( $85 \pm 9.35$ vs $78 \pm 11.58$ years, $\mathrm{p}<0.001)$, more likely to be admitted for pneumonia ( $22 \%$ vs. $4.9 \%$ of those with lower Fls; $p<0,001)$. They also had more comorbidity, loss of autonomy, cognitive impairment, social frailty, and nursing care needs. The subgroup with FIs $\geq 17$ had significantly higher in-hospital mortality ( $30.28 \%$ vs $6.25 \%, p<0.001)$, longer hospital stay ( 13 vs. 10 days, $p<0.05$ ), and higher rates of discharge to nursing homes.

Conclusions: Evaluation of internal medicine patients with the Flugelman Index may be helpful for identifying more critical patients likely to require longer hospitalization and to detect factors affecting the hospital stay. This information can be useful for more effective discharge planning. (c) 2010 Elsevier Srl. All rights reserved.
\end{abstract}

* Corrispondenza: Podere Alberese, Località Montecalvoli 41 - 53041 Asciano (SI).

E-mail: chiara.bozzano@virgilio.it (C. Bozzano). 


\section{Introduzione}

A causa del progressivo invecchiamento demografico e dell'impatto delle patologie croniche sul sistema sanitario, la Medicina Interna è costretta a confrontarsi con il complex adaptive system [1], caratterizzato da un framework di problemi sociosanitari e non dalla tradizionale relazione causa-effetto. Il malato internistico è più frequentemente anziano, pluripatologico, disabile o fragile, in politerapia farmacologica, in molti casi critico e con elevato rischio di danno iatrogeno e complicanze.

Parallelamente alla complessità multisistemica del paziente, emerge il problema delle "dimissioni difficili" $[2,3]$, che sta inducendo alla sperimentazione di nuovi modelli di gestione della cronicità [4-8]. D'altronde è noto come l'adeguata pianificazione della dimissione attraverso l'analisi sistematica dei pazienti anziani [9] eserciti un impatto positivo sul sistema, anche per modeste riduzioni della durata di ricovero e delle riammissioni ospedaliere [10].

In questo panorama, la valutazione multidimensionale (VMD) può offrire validi strumenti per identificare, descrivere e ponderare i molteplici problemi che investono l'anziano [11-14]: la metodica è analitica e multidisciplinare [11] e consente di effettuare una valutazione olistica della persona [13]. Dal punto di vista operativo si attua somministrando al paziente scale di valutazione ampiamente validate in letteratura. Tra queste, per esempio, il Katz Index of Independence in Activities of Daily Living [15] e il Barthel Index [16] (per indagare le capacità funzionali), il Mini-Mental State Examination [17], il Short Portable Mental Status Questionnaire [18], il Neuropsychiatric Inventory [19] e la Geriatric Depression Scale [20,21] (per la componente cognitivo-comportamentale e il tono dell'umore), il Mini Nutritional Assessment [22] (per lo stato nutrizionale), la Cumulative Illness Rating Scale [23] e l'indice di comorbilità geriatrica (per il grado di comorbilità), la Waterlow Scale [24] e la Braden Scale [25] (per il rischio di lesioni da decubito).

A oggi, tuttavia, è scarsa la letteratura inerente a scale di valutazione per individuare pazienti complessi e di difficile dimissione ospedaliera [26,27]. In merito a questo spinoso problema, proponiamo l'indice di Flugelman (IF) [28], validato alla fine degli anni Ottanta: lo studio osservazionale dimostrava come un punteggio complessivo $\geq 17$ consentisse di individuare pazienti a maggior rischio di ricovero in struttura e mortalità a 3 mesi.

\section{Materiali e metodi}

Dal $1^{\circ}$ settembre 2007 al 31 ottobre 2007 sono stati reclutati tutti i pazienti ricoverati consecutivamente presso i reparti di Medicina Interna e Medicina Interna e Geriatria dell'Ospedale San Donato di Arezzo. Sono stati esclusi i pazienti che rifiutavano di partecipare allo studio e coloro che erano deceduti o venivano dimessi entro 24 ore dall'ingresso in reparto.

Per coloro che hanno aderito allo studio è stato raccolto, da parte di personale medico e infermieristico opportunamente addestrato, un pool di informazioni demografiche, cliniche e sociali (entro le 48 ore successive al ricovero). Tra le variabili considerate: età, sesso, condizione sociale, dati clinici (motivo del ricovero, grado di comorbilità), capacità funzionali prima del ricovero e alla dimissione, presenza di lesioni da decubito, eventuale incontinenza urinaria e/o fecale o uso di catetere vescicale (CV), necessità di assistenza nell'alimentazione, presenza di sondino nasogastrico (SNG) o gastrostomia percutanea (PEG).

Nell'ottica della VMD sono state applicati l'IF, l'indice di comorbilità geriatrica e la Numeric Intensity Scale (NIS), quest'ultima per la valutazione del dolore percepito. L'indice di Barthel per le capacità funzionali di base nella vita quotidiana è stato calcolato in riferimento alle performance fisiche prima dell'evento acuto (a domicilio) e in fase di predimissione (nelle 24 ore precedenti la dimissione).

Attraverso l'analisi a posteriori della Scheda di Dimissione Ospedaliera (SDO) sono state inoltre registrate la durata della degenza, la diagnosi (primaria e secondaria) e la modalità di dimissione.

Lo studio è stato condotto previa approvazione da parte del Comitato Etico locale e con l'acquisizione del consenso informato di ciascun paziente osservato nello studio.

\section{Analisi statistica}

I risultati descrittivi e della popolazione sono espressi in valore medio, deviazione standard e proporzioni. Nel confronto fra medie, il grado di significatività è stato ottenuto mediante il test $\mathrm{t}$ di Student. Il test di associazione $\mathrm{Chi}^{2}$ è stato usato per confrontare le proporzioni.

\section{Risultati}

Sono stati reclutati complessivamente 413 pazienti con età media di $80 \pm 11,37$ anni (56,1\% dei quali donne) (tabella 1).

Tabella 1 Caratteristiche della popolazione.

\begin{tabular}{|c|c|c|c|c|c|c|c|c|c|c|}
\hline & \multicolumn{3}{|c|}{ Popolazione totale $\mathrm{N}=413$} & \multicolumn{3}{|c|}{ IF $<17 \mathrm{~N}=304$} & \multicolumn{3}{|c|}{ IF $\geq 17 \mathrm{~N}=109$} & \multirow[t]{2}{*}{$\mathrm{p}$} \\
\hline & (\%) & Media \pm DS & Range & (\%) & Media \pm DS & Range & (\%) & Media \pm DS & Range & \\
\hline Donne & 56,1 & & & 53,2 & & & 63,3 & & & NS \\
\hline Età (anni) & & $80 \pm 11,37$ & & & $78 \pm 11,58$ & & & $85 \pm 9,35$ & & $<0,001$ \\
\hline$<65$ & 8,8 & & & 11,1 & & & 1,8 & & & \\
\hline $65-74$ & 17,6 & & & 19,2 & & & 13,8 & & & \\
\hline $75-84$ & 32,7 & & & 35,8 & & & 23,9 & & & \\
\hline$\geq 85$ & 40,9 & & & 33,9 & & & 60,5 & & & \\
\hline
\end{tabular}

Legenda: IF = indice di Flugelman; DS = deviazione standard. 
In base alla valutazione mediante IF, 109 pazienti totalizzavano uno score $\geq 17$. In questo gruppo l'età media era significativamente superiore rispetto al gruppo con score $<17(85 \pm 9,35$ anni vs $78 \pm 11,58 ; p<0,001)$.

In fig. 1 sono rappresentate le principali cause di ricovero, come estrapolato dall'analisi delle SDO.

Confrontando le due popolazioni suddivise in base all'IF, e fatta eccezione per "Altre cause di ricovero", il motivo principale di ricovero ospedaliero era un evento di polmonite o broncopolmonite nei soggetti con score IF $\geq 17$ (15/304, $4,9 \%$, vs $24 / 109,22 \%$; $p<0,001$ ) e un episodio di scompenso cardiaco acuto nei soggetti con IF $<17$ (62/304, 20\%, vs $13 /$ $109,11,9 \% ; p<0,05)$. Anche la demenza era maggiormente incidente nel gruppo con punteggio IF $\geq 17$, anche se in modo statisticamente non significativo. Tuttavia, quando la patologia veniva suddivisa per diagnosi primaria e secondaria, la demenza e/o il declino cognitivo erano significativamente superiori nella popolazione con IF $\geq 17$ rispetto al gruppo di controllo $(81 / 109,74,3 \%$, vs $77 / 304,25,3 \%$; $p<0,001)$.

La complessità del paziente è stata indagata attraverso parametri di tipo medico, infermieristico e sociale, come esplicitato in tabella 2 . I pazienti con IF $\geq 17$ avevano un grado di comorbilità più elevato $(78 / 109,71,5 \%$, vs $106 / 304$, $34,8 \% ; p<0,001)$ e una degenza media significativamente superiore $(13 \pm 9,92$ giorni vs $10 \pm 8,64 ; p<0,05)$. Inoltre presentavano maggiore "fragilità sociale", intesa come condizione in cui la persona fosse priva di sostegno familiare adeguato o provenisse da struttura residenziale $(24 / 109$, $22 \%$, vs $13 / 304,4,2 \% ; p<0,001)$. Tra gli indicatori di complessità infermieristica, si noti come il gruppo con indice prognostico più elevato fosse significativamente più incontinente o portatore di catetere, oltre che più frequentemente allettato e con lesioni da pressione rispetto al gruppo di controllo. Questi pazienti, inoltre, erano incapaci ad alimentarsi in modo del tutto autonomo ed erano portatori di SNG o PEG con frequenza significativamente superiore.

Se si considerano i pazienti vivi alla dimissione, in Fig. 2 è rappresentata la popolazione suddivisa in base all'IF e stratificata in relazione al punteggio ottenuto con l'indice di Barthel per le capacità funzionali nella vita quotidiana. I soggetti con IF $\geq 17$ mostravano maggiore compromissione dell'autonomia già prima del ricovero (score $\leq 49: 67 / 76$ vs $50 / 284 ; p<0,001$ ) e in fase di predimissione la quasi totalità dei pazienti valutati necessitava di completa assistenza nelle

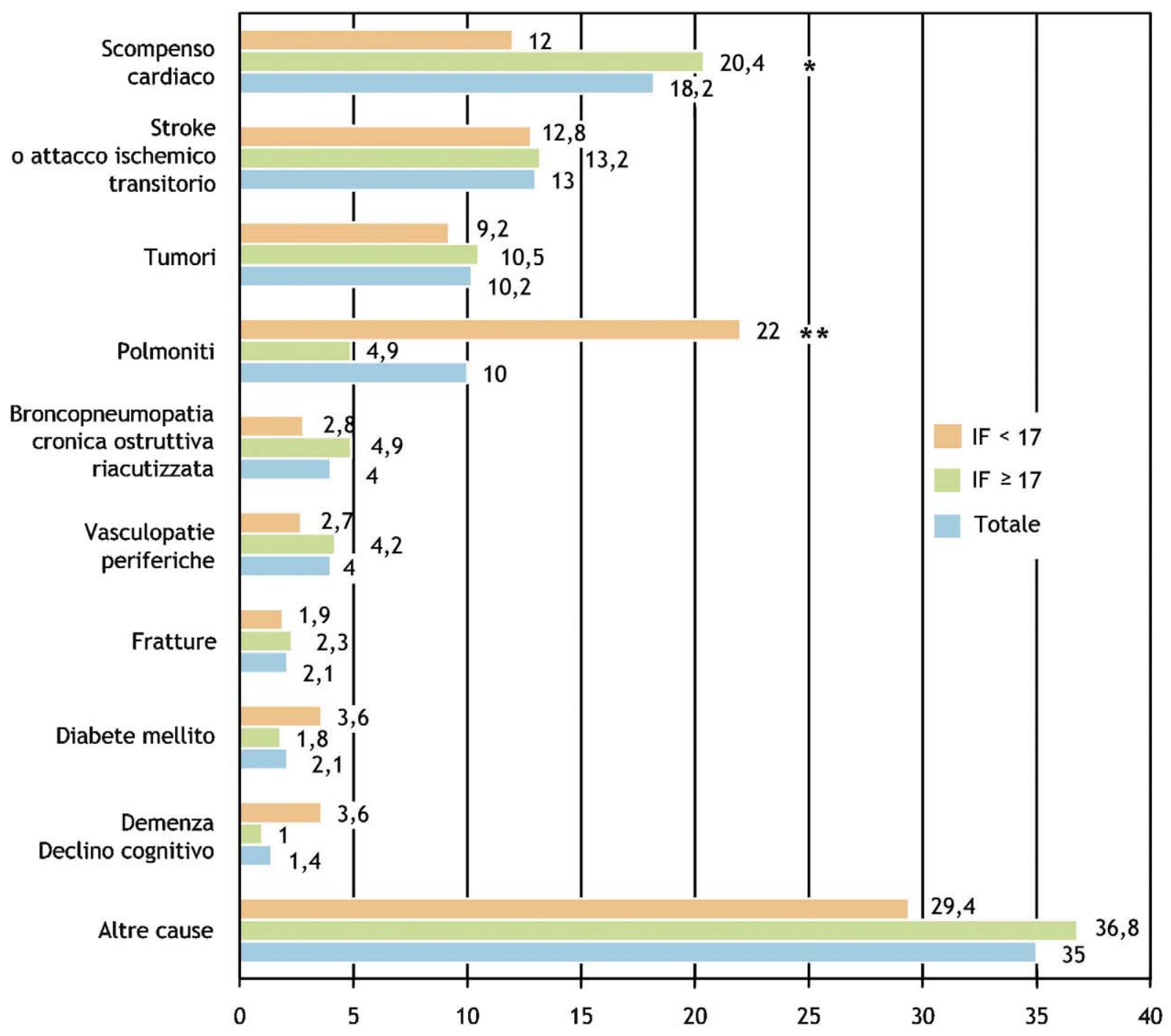

Figura 1 Diagnosi di dimissione. 
Tabella 2 Parametri di complessità.

\begin{tabular}{|c|c|c|c|c|}
\hline & $\begin{array}{l}\text { Popolazione totale } \\
\qquad(\mathrm{N}=413)\end{array}$ & $\begin{array}{c}\mathrm{IF}<17 \\
(\mathrm{~N}=304)\end{array}$ & $\begin{array}{l}\text { IF } \geq 17 \\
(N=109)\end{array}$ & $\mathrm{p}$ \\
\hline Giornate di degenza & $11 \pm 9,11$ & $10 \pm 8,64$ & $13 \pm 9,92$ & $<0,05$ \\
\hline \multicolumn{5}{|l|}{ Indice di comorbilità geriatrica (\%) } \\
\hline Grado I & 0,6 & 0,7 & 0 & \\
\hline Grado II & 11,3 & 14,8 & 1,8 & \\
\hline Grado II & 43,6 & 49,7 & 26,7 & \\
\hline Grado IV & 44,5 & 34,8 & 71,5 & $<0,001$ \\
\hline Compromissione delle funzioni cognitive (\%) & 12,3 & 7,5 & 25 & $<0,001$ \\
\hline Fragilità sociale $(\%)$ & 8,9 & 4,27 & 22 & $<0,001$ \\
\hline Incontinenza, presenza di catetere (\%) & 46,00 & 26,64 & 100,00 & $<0,001$ \\
\hline Allettamento (\%) & 26,8 & 26,6 & 75,2 & $<0,001$ \\
\hline Lesioni da pressione (\%) & 14 & 5,2 & 41 & $<0,001$ \\
\hline Bisogno di assistenza durante il pasto & 33,6 & 18,7 & 72,4 & $<0,001$ \\
\hline Sondino nasogastrico o gastrostomia percutanea (\%) & 7,7 & 0,9 & 26,6 & $<0,001$ \\
\hline
\end{tabular}

attività della vita quotidiana (74/76 vs 55/284; $p<0,001)$. In accordo con la letteratura, la popolazione con IF $\geq 17$ aveva anche prognosi peggiore a breve termine con mortalità intraospedaliera significativamente superiore rispetto al gruppo di controllo (33/109 vs 19/304, p <0,001) (fig. 3). Inoltre, considerando la modalità di dimissione quale indicatore indiretto di complessità, i pazienti con indice prognostico più elevato richiedevano più frequentemente la dimissione in modalità protetta, in termini di invio in Ospedale di Comunità $(6 / 109$ vs $3 / 304, p<0,001)$ o Residenza Sanitaria Assistenziale (12/109 vs 5/304; p < 0,001). La popolazione con IF $<17$, al contrario, veniva dimessa in modo ordinario in una percentuale significativamente superiore $(261 / 304$ vs $54 / 109 ; p<0,001)$.

\section{Discussione}

Come raccomandato dalla European Federation of Internal Medicine [29], il medico di Medicina Interna deve saper rivestire molteplici funzioni (diagnostiche, terapeutiche,

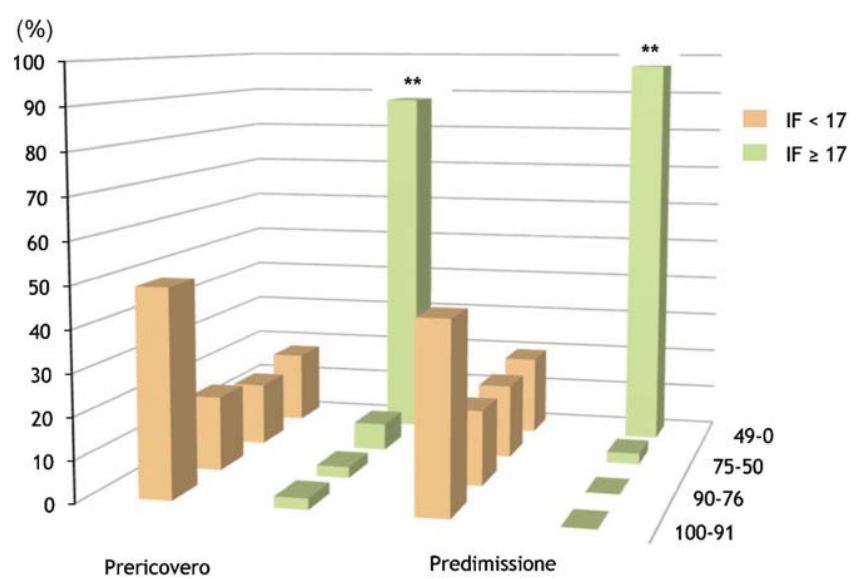

Figura 2 Capacità funzionali prima e dopo l'evento acuto. di counselling, educative, gestionali ecc.) e valutare in modo olistico il paziente considerandone le condizioni cliniche, le capacità funzionali e cognitive, la condizione sociale, la compliance alla terapia. Il grado di comorbilità, infatti, crea problemi diagnostico-terapeutici [30] ma da solo non è sufficiente a spiegare la complessità del malato [31].

La popolazione reclutata nello studio sembra rappresentare fedelmente quanto sperimentiamo quotidianamente nei nostri reparti, dal momento che l'età media complessiva è tendenzialmente elevata. Benché "invecchiamento" non sia sinonimo di "malattia", è pur vero che l'età predispone di per sé a problematiche che rendono l'anziano potenzialmente complesso, se non addirittura fragile. La popolazione con punteggio IF $\geq 17$, rispetto al gruppo di controllo, soddisfa pienamente i recenti criteri per la diagnosi di questa sindrome geriatrica [32]: ha età media $>80$ anni, capacità funzionali e deambulazione compromesse, presenta alta prevalenza di allettati, di incontinenti e di pazienti con declino cognitivo o demenza. Lo stato nutrizionale è gravemente compromesso e tale aspetto, associato al maggior allettamento, contribuirebbe a spiegare il maggior rischio

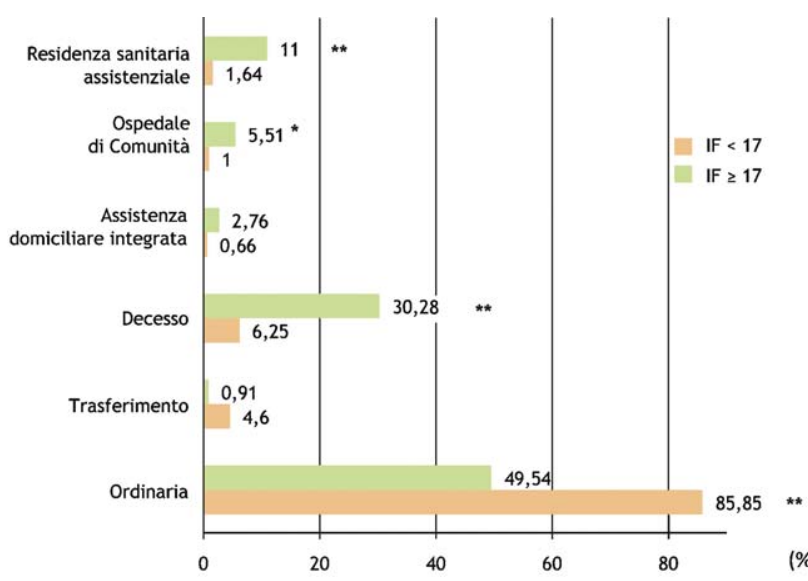

Figura 3 Modalità di dimissione (dati SDO). 
di lesioni da decubito e la maggiore incidenza di polmonite quale motivo del ricovero ospedaliero. L'embricarsi di questi fattori, associati al grado di comorbilità, renderebbe ragione dell'elevata degenza media.

È noto, inoltre, che l'ospedalizzazione di un anziano può far precipitare la disabilità nelle attività della vita quotidiana [33], determinando gravi ripercussioni per il paziente e la sua famiglia e peggiorando la prognosi a breve e medio termine $[34,35]$. Nella popolazione studiata, i pazienti con IF $\geq 17$ mostravano disabilità medio-grave già prima dell'evento acuto, avvenuto il quale la disabilità peggiorava ulteriormente come segno di ridotta riserva funzionale. In accordo con la letteratura, anche nel nostro studio la casistica con significativo peggioramento funzionale correla con una prognosi peggiore a breve termine, come dimostrato dalla maggiore probabilità di decesso ospedaliero nel gruppo con IF $\geq$ 17 nonché, a 4 mesi, dall'alta percentuale di decessi (38\%) e di re-ricoveri (63\%).

A confermare indirettamente la difficoltà incontrata in fase di dimissione nei pazienti con IF $\geq 17$, si osservi il maggior ricorso alle risorse territoriali (Ospedale di Comunità e Residenza Sanitaria Assistenziale): si tratta di anziani che, per la loro condizione di fragilità e complessità sociosanitaria e assistenziale, richiedono la progettazione di un percorso postospedaliero finalizzato alla continuità assistenziale e alla presa in carico da parte del territorio.

\section{Conclusioni}

Com'è noto, la Medicina Interna si confronta ormai da tempo con la complessità del paziente anziano. Tra gli obiettivi della "best clinical practice" dovremmo promuovere l'implementazione delle nostre competenze con la metodologia della valutazione multidimensionale geriatrica. Ideata per affrontare la complessità, fornisce strumenti che coniugano rapidità di esecuzione e buon livello di sensibilità, permettendo di far luce sulle criticità proprie dell'anziano. Con il nostro studio intendiamo proporre l'indice di Flugelman quale scala di valutazione che potrebbe individuare i pazienti complessi e la cui dimissione potrebbe essere più difficile dell'atteso. $\grave{E}$ evidente che, se utilizzato nelle prime fasi del ricovero ospedaliero, aiuterebbe il medico a pianificare anche la fase di dimissione.

\section{Conflitto di interesse}

Gli autori dichiarano di essere esenti da conflitto di interessi.

\section{Ringraziamenti}

Gli autori ringraziano la Fondazione Cesalpino e tutto il personale infermieristico che ha permesso lo svolgimento accurato dello studio.

\section{Bibliografia}

[1] Pisek P. Redesigning health care with insights from the science of complex adaptive systems. In: Committee on Quality Health Care in America, Institute of Medicine. Crossing the Quality Chasm: A new health system for the $21^{\text {st }}$ Century. Washington,
DC: National Academy Press, National Academy of Sciences; 2001. pp. 309-22.

[2] Nardi R, Scanelli G, Tragnone A, Lolli A, Kalfus P, Baldini A, et al. Difficult hospital discharge in internal medicine wards. Intern Emerg Med 2007 Jun;2(2):95-9.

[3] Pedace C. Difficult hospital discharges and disease management. Intern Emerg Med 2007;2(2):74.

[4] Coleman K, Austin BT, Brach C, Wagner EH. Evidence on the Chronic Care Model in the new millennium. Health Aff (Millwood) $2009 ; 28(1): 75-85$.

[5] Melis RJ, Olde Rikkert MG, Parker SG, van Eijken MI. What is intermediate care? BMJ 2004;329(7462):360-1.

[6] Naylor M, Keating SA. Transitional care. Am J Nurs 2008;108(9 Suppl):58-63.

[7] Jonker AA, Comijs HC, Knipscheer KC, Deeg DJ. Promotion of self-management in vulnerable older people: a narrative literature review of outcomes of the Chronic Disease Self-Management Program (CDSMP). Eur J Ageing 2009;6(4):303-14.

[8] Ofman JJ, Badamgarav E, Henning JM, Knight K, Gano Jr AD, Levan RK, et al. Does disease management improve clinical and economic outcomes in patients with chronic diseases? A systematic review. Am J Med 2004 Aug 1;117(3):182-92. Review.

[9] Rubenstein LZ, Josephson KR, Wieland GD, English PA, Sayre JA, Kane RL. Effectiveness of a geriatric evaluation unit. A randomized clinical trial. N Engl J Med 1984;311(26):1664-70.

[10] Shepperd S, Parkes J, McClaren J, Phillips C. Discharge planning from hospital to home. Cochrane Database Syst Rev 2004; (1):CD000313.

[11] Rubenstein L. The clinical effectiveness of multidimensional geriatric assessment. J Am Geriatr Soc 1983;31(12):758-62.

[12] Stuck AE, Siu AL, Wieland GD, Adams J, Rubenstein LZ. Comprehensive geriatric assessment: a meta-analysis of controlled trials. Lancet 1993;342(8878):1032-6.

[13] Health and Public Policy Committee. American College of Physicians. Comprehensive functional assessment for elderly patients. Ann Intern Med 1988;109(1):70-2.

[14] Reuben DB, Solomon DH. Assessment in geriatrics. Of caveats and names. J Am Geriatr Soc 1989;37(6):570-2.

[15] Wallace M, Shelkey M, Hartford Institute for Geriatric Nursing. Katz Index of Independence in Activities of Daily Living (ADL). Urol Nurs 2007;27(1):93-4.

[16] Shah S, Vanclay F, Cooper B. Improving the sensitivity of the Barthel Index for stroke rehabilitation. J Clin Epidemiol 1989;42(8):703-9.

[17] Hensel A, Angermeyer MC, Riedel-Heller SG. Measuring cognitive change in older adults: reliable change indices for the MiniMental State Examination. J Neurol Neurosurg Psychiatry 2007;78(12):1298-303.

[18] Erkinjuntti T, Sulkava R, Wikström J, Autio L. Short Portable Mental Status Questionnaire as a screening test for dementia and delirium among the elderly. J Am Geriatr Soc 1987;35(5): 412-6.

[19] Cummings JL, Mega M, Gray K, Rosenberg-Thompson S, Carusi DA, Gornbein J. The Neuropsychiatric Inventory: comprehensive assessment of psychopathology in dementia. Neurology 1994;44(12):2308-14.

[20] Montorio I, Izal M. The Geriatric Depression Scale: a review of its development and utility. Int Psychogeriatr 1996;8(1):103-12.

[21] Greenberg SA. How to try this: the Geriatric Depression Scale: Short Form. Am J Nurs 2007;107(10):60-9.

[22] Guigoz Y, Lauque S, Vellas BJ. Identifying the elderly at risk for malnutrition. The Mini Nutritional Assessment. Clin Geriatr Med 2002;18(4):737-57.

[23] Salvi F, Miller MD, Grilli A, Giorgi R, Towers AL, Morichi V, et al. A manual of guidelines to score the modified comulative illness rating scale and its validation in acute hospitalized elderly patients. J Am Geriatr Soc 2008 Oct;56(10):1926-31. 
[24] Serpa LF, de Gouveia Santos VL, Gomboski G, Rosado SM. Predictive validity of Waterlow Scale for pressure ulcer development risk in hospitalized patients. J Wound Ostomy Continence Nurs 2009;36(6):640-6.

[25] Kring DL. Reliability and validity of the Braden Scale for predicting pressure ulcer risk. J Wound Ostomy Continence Nurs 2007;34(4):399-406.

[26] Mistiaen P, Duijnhouwer E, Prins-Hoekstra A, Ros W, Blaylock A. Predictive validity of the BRASS index in screening patients with post-discharge problems. Blaylock Risk Assessment Screening Score. J Adv Nurs 1999;30(5):1050-6.

[27] Nardi R, Scanelli G, Borioni D, Grandi M, Sacchetti C, Parenti M, et al. The assessment of complexity in internal medicine patients. The FADOI Medicomplex Study. Eur J Intern Med 2007 Jul;18(4):283-7.

[28] Flugelman MY, Ben David Y, Harats N, Eliakim M. A simple prognostic index for hospitalized geriatric patients. A prospective study of 70 patients. Gerontology 1986;32(5):272-6.

[29] Palsson R, Kellett J, Lindgren S, Merino J, Semple C, Sereni D, For the EFIM/UEMS Working Group on Competencies in Internal Medicine in Europe. Core competencies of the European internist: A discussion paper. Eur J Intern Med 2007;18(2):104-8.
[30] Wade DT, Halligan PW. Do biomedical models of illness make for good healthcare systems? BMJ 2004;329(7479): 1398-401.

[31] Nardi R, Scanelli G, Corrao S, lori I, Mathieu G, Cataldi Amatrian R. Co-morbidity does not reflect complexity in internal medicine patients. Eur J Intern Med 2007;18(5):359-68.

[32] Abellan van Kan G, Rolland Y, Bergman H, Morley JE, Kritchevsky SB, Vellas B. The IANA Task Force on frailty assessment of older people in clinical practice. J Nutr Health Aging 2008;12(1): 29-37.

[33] Boyd CM, Landefeld CS, Counsell SR, Palmer RM, Fortinsky RH, Kresevic $D$, et al. Recovery of activities of daily living in older adults after hospitalization for acute medical illness. J Am Geriatr Soc 2008 Dec;56(12):2171-9.

[34] Baztán JJ, Gálvez CP, Socorro A. Recovery of functional impairment after acute illness and mortality: one-year follow-up study. Gerontology 2009;55(3):269-74.

[35] Covinsky KE, Palmer RM, Fortinsky RH, Counsell SR, Stewart AL, Kresevic $D$, et al. Loss of independence in activities of daily living in older adults hospitalized with medical illnesses: increased vulnerability with age. J Am Geriatr Soc 2003 Apr;51(4): 451-8. 